\section{Assessment of the additional clinical potential of X-ray dark-field imaging for breast cancer in a preclinical setup}

\author{
Julius Emons, Peter A. Fasching, Marius Wunderle, Felix Heindl, Jens Rieger, Florian Horn, \\ Georg Pelzer, Andre Ritter, Thomas Weber, Marcus Radicke, Iris Polifka, David L. Wachter, \\ Evelyn Wenkel, Thilo Michel, Michael Uder, Arndt Hartmann, Gisela Anton, Matthias W. \\ Beckmann, Rüdiger Schulz-Wendtland and Sebastian M. Jud
}

\begin{abstract}
Background: Mammography can identify calcifications up to $50-100 \mu \mathrm{m}$ in size as a surrogate parameter for breast cancer or ductal carcinoma in situ (DCIS). Microcalcifications measuring $<50 \mu \mathrm{m}$ are also associated with breast cancer or DCIS and are frequently not detected on mammography, although they can be detected with dark-field imaging. This study examined whether additional breast examination using $X$-ray dark-field imaging can increase the detection rate of calcifications. Advances in knowledge:

(1) evaluation of additional modality of breast imaging;

(2) specific evaluation of breast calcifications.

Implications for patient care: the addition of X-ray dark-field imaging to conventional mammography could detect additional calcifications.
\end{abstract}

Methods: Talbot-Lau X-ray phase-contrast imaging and X-ray dark-field imaging were used to acquire images of breast specimens. The radiation dosage with the technique is comparable with conventional mammography. Three X-ray gratings with periods of $5-10 \mu \mathrm{m}$ between the $X$-ray tube and the flat-panel detector provide three different images in a single sequence: the conventional attenuation image, differential phase image, and dark-field image. The images were read by radiologists. Radiological findings were marked and examined pathologically. The results were described in a descriptive manner.

Results: A total of 81 breast specimens were investigated with the two methods; 199 significant structures were processed pathologically, consisting of 123 benign and 76 malignant lesions (DCIS or invasive breast cancer). X-ray dark-field imaging identified 15 additional histologically confirmed carcinoma lesions that were visible but not declared suspicious on digital mammography alone. Another four malignant lesions that were not visible on mammography were exclusively detected with $\mathrm{X}$-ray dark-field imaging.

Conclusions: Adding X-ray dark-field imaging to digital mammography increases the detection rate for breast cancer and DCIS associated lesions with micrometer-sized calcifications. The use of X-ray dark-field imaging may be able to provide more accurate and detailed radiological classification of suspicious breast lesions.

Adding X-ray dark-field imaging to mammography may be able to increase the detection rate and improve preoperative planning in deciding between mastectomy or breast-conserving therapy, particularly in patients with invasive lobular breast cancer.

Keywords: breast cancer, interferometry, microcalcifications, mammography
Ther Adv Med Oncol

2020, Vol. 12: 1-10

DOI: $10.1177 /$

1758835920957932

(c) The Author(s), 2020. Article reuse guidelines: sagepub.com/journalspermissions
Correspondence to: Peter A. Fasching Department of Gynecology and Obstetrics, Erlangen University Hospital, Comprehensive Cancer Center Erlangen-EMN

Universitätsstrasse 21-23, Erlangen 91054, Germany peter.faschinglukerlangen.de

Julius Emons

Marius Wunderle

Felix Heindl

Matthias W. Beckmann

Sebastian M. Jud

Department of Gynecology

and Obstetrics, Friedrich Alexander University of

Erlangen-Nuremberg.

Erlangen, Bayern,

Germany

Jens Rieger Florian Horn Georg Pelzer Andre Ritter

Thomas Weber

Thilo Michel

Gisela Anton

Erlangen Center for Astroparticle Physics,

Friedrich Alexander

University of Erlangen-

Nuremberg, Erlangen,

Germany

Marcus Radicke

Siemens Healthcare

$\mathrm{GmbH}$, Erlangen, Germany

Iris Polifka

David L. Wachter

Arndt Hartmann

Institute of Pathology,

Erlangen University

Hospital, Erlangen,

Germany

Evelyn Wenkel

Michael Uder

Rüdiger Schulz-

Wendtland

Institute of Diagnostic

Radiology, Erlangen

University Hospital,

Erlangen, Bayern,

Germany 


\section{Introduction}

One of the principal strategies for reducing the mortality rate for patients with breast cancer involves screening mammography and early detection, and the importance of mammography for screening is underlined in recent guidelines. ${ }^{1-3}$ Several attempts have been made to increase the sensitivity of breast imaging without reducing its specificity, with varying degrees of success; for example, using full-field mammography instead of screen-film mammography, or adding ultrasound and magnetic resonance imaging. ${ }^{2,4,5}$

Microcalcifications on mammography images can be an important indicator of breast cancer. Patients with these lesions are therefore recommended to undergo further histological clarification. However, the majority of these lesions turn out to be benign, so that additional interventional diagnostic procedures could have been avoided. ${ }^{6-8}$

Mammography is able to detect microcalcifications at least $50-100 \mu \mathrm{m}$ in size. ${ }^{9}$ Conventional mammography is based on the attenuation of $\mathrm{X}$-ray radiation by different tissues. The amount of attenuation is dependent on the absorption coefficient of the respective tissue, which increases with greater tissue density. Because differences between several types of soft-tissue decline with increasing X-ray energy, conventional mammography is performed at lower tube voltages in comparison with other clinically relevant X-ray procedures. ${ }^{9}$ Major limitations of mammography are its reduced sensitivity and specificity in certain populations. The sensitivity can be as high as $90 \%,{ }^{10}$ but may be up to $50 \%$ lower in women who are young, those receiving hormone replacement therapy, or those who have higher-density breast tissue ${ }^{11,12}$

Smaller microcalcifications $<50 \mu \mathrm{m}$ in size cannot be adequately detected by mammography. However, it has been shown that imaging with X-ray Talbot-Lau interferometry can identify such microcalcifications in a preclinical setup at non-clinical radiation dose. ${ }^{13,14}$ Interferometric imaging set-ups for X-ray Talbot-Lau interferometry typically consist of three $\mathrm{X}$-ray gratings with period lengths in the range of $2-10 \mu \mathrm{m}$, which are placed between the X-ray tube and the flat-panel detector. The combination of these gratings delivers three different images in a single imaging sequence. The combination of the gratings, the acquisition scheme, and image reconstruction provides an absorption image, a differential phase image, and a dark-field image from one imaging sequence. The dark-field image is of particular interest for small structures such as microcalcifications smaller than $50 \mu \mathrm{m} .{ }^{13-16}$

The aim of the present study was to investigate whether additional X-ray dark-field imaging can increase the detection rate of microcalcifications and consequently of breast cancer in comparison with conventional digital mammography alone.

\section{Materials and methods}

\section{Patients}

Patients were recruited from the University Breast Center at Erlangen University Hospital. The patients were treated in accordance with standard clinical care. They were eligible for inclusion in the study if they were at least 18-years old and had undergone either mastectomy or breast-conserving therapy. The reasons for definitive breast surgery with mastectomy or breast-conserving therapy included histologically confirmed ductal carcinoma in situ (DCIS) or invasive breast cancer; a second resection following a first resection with residual tumor; or risk-reducing mastectomy in patients who were at increased risk; or orthopedic reasons. All the patients provided written, informed consent for the procedures in the study and for the scientific use of their data. The study was approved by the ethics committee of the medical faculty of the University of ErlangenNuremberg (ID: Re. no. 4514).

\section{Clinical data}

The patients included in this trial were taking part in the i-MODE-B (Imaging and Molecular Detection-Breast) study. The patient data were recorded prospectively. The clinical data were documented from the patients' charts into an electronic case-report form. All patient and tumor characteristics were documented, monitored, and audited as part of the certification processes required by the German Cancer Society (Deutsche Krebsgesellschaft) and by the German Society for Mastology (Deutsche Gesellschaft fuer Senologie e.V.). ${ }^{17,18}$

\section{Study procedures and imaging data}

The participants in the study initially underwent breast diagnostic procedures, including a standard mammography and ultrasonography. After 
surgery, secondary imaging of the resected breast tissue was carried out, including digital mammography and X-ray dark-field imaging. Firstly, the breast specimen was fixed from anterior and posterior using a custom-made Perspex plate to ensure reproducibility of the areas of interest in the imaging process. Conventional mammography as performed in routine clinical work was carried out at the Institute of Diagnostic Radiology at Erlangen University Hospital. The fixed specimens were then transferred to the Erlangen Center for Astroparticle Physics, where the X-ray dark-field imaging was performed.

Imaging of the breast specimens was carried out in the anterior-posterior view with both imaging techniques. The dark-field images were processed using inversion and adjustment of contrast and brightness by blinded non-medical staff. This process was necessary due to the missing processing algorithm normally applied to diagnostic mammography images. Both types of image were only used for scientific purposes and were analyzed at a diagnostic workstation using a clinically available picture archiving and communication system. The mammographic images were read and classified based on the Breast Imaging-Reporting and Data System (BI-RADS) criteria by two radiologists with experience in breast imaging.

All lesions that were visible on X-ray dark-field imaging were marked. The BI-RADS classification was only applied to the mammographic images, since criteria for the BI-RADS classification have not yet been established for X-ray darkfield imaging. If additional suspicious lesions were detected with mammography alone, these were marked and classified. Suspicious lesions were classified as $\geqslant$ BI-RADS 4 . Finally, all the lesions that were visible on X-ray dark-field imaging and/or all suspicious lesions on mammography were assessed pathologically (Figure 1). After the two imaging techniques had been carried out, a web-based interdisciplinary conference was held that included radiologists, physicists, gynecologists, and pathologists. The mammographic and X-ray dark-field images were merged together, and marked lesions were discussed on an interdisciplinary basis to select those that required further pathological assessment. The selected lesions from both imaging techniques were marked with a circle on the mammogram. These markings were projected onto the breast specimen at actual size for further pathological assessment.

\section{Mammographic imaging}

The digital mammographic images were obtained using a MAMMOMAT Inspiration device (Siemens AG, Erlangen, Germany) with a physical pixel size of $85 \mu \mathrm{m}$. Digital mammography is able to detect microcalcifications at least $50-100 \mu \mathrm{m}$ in size, as previously described.

\section{$X$-ray dark-field imaging}

Grating-based X-ray dark-field imaging is a method of phase-contrast imaging that provides additional information from absorption and from the X-ray dark-field signal. X-ray phase-contrast imaging (XPCI) is a new technique based on the appearance of a phase shift when $\mathrm{X}$-ray radiation crosses the border between different tissues with varying refraction indices. The effect of the phase shift in phase-contrast imaging is greater than the effect of absorption in conventional mammographic imaging, at the energy level that is used for conventional mammography $(20-30 \mathrm{kV}) \cdot{ }^{13-16}$ Phase-contrast imaging has been reported to increase soft-tissue contrast and to be able to demonstrate extremely small structures at high resolution. ${ }^{19}$

Various techniques for phase-contrast imaging have so far been investigated, one of which is grating interferometry. When three X-ray gratings with periods in the order of a few micrometers between the X-ray tube and the flat-panel detector are used, it is possible to acquire three different images in a single image sequence: the conventional attenuation image, the differential phase image, and the X-ray dark-field image. The X-ray dark-field image is caused by scattering of X-ray radiation and is capable of detecting microcalcifications at high resolution. ${ }^{13,14}$

\section{Pathology data}

The pathologists were provided with the fixed breast specimen and an actual-size transparent print-out containing the marked lesions of interest. The print-out was placed on top of the fixed breast specimen. A standard syringe needle was used to re-identify the two-dimensional findings in the breast tissue (one to a maximum of seven per breast specimen). The marked areas were excised and sliced into $3 \mathrm{~mm}$ thick sections. The tissue was fully embedded, stained, and assessed in accordance with standard pathological procedures. 


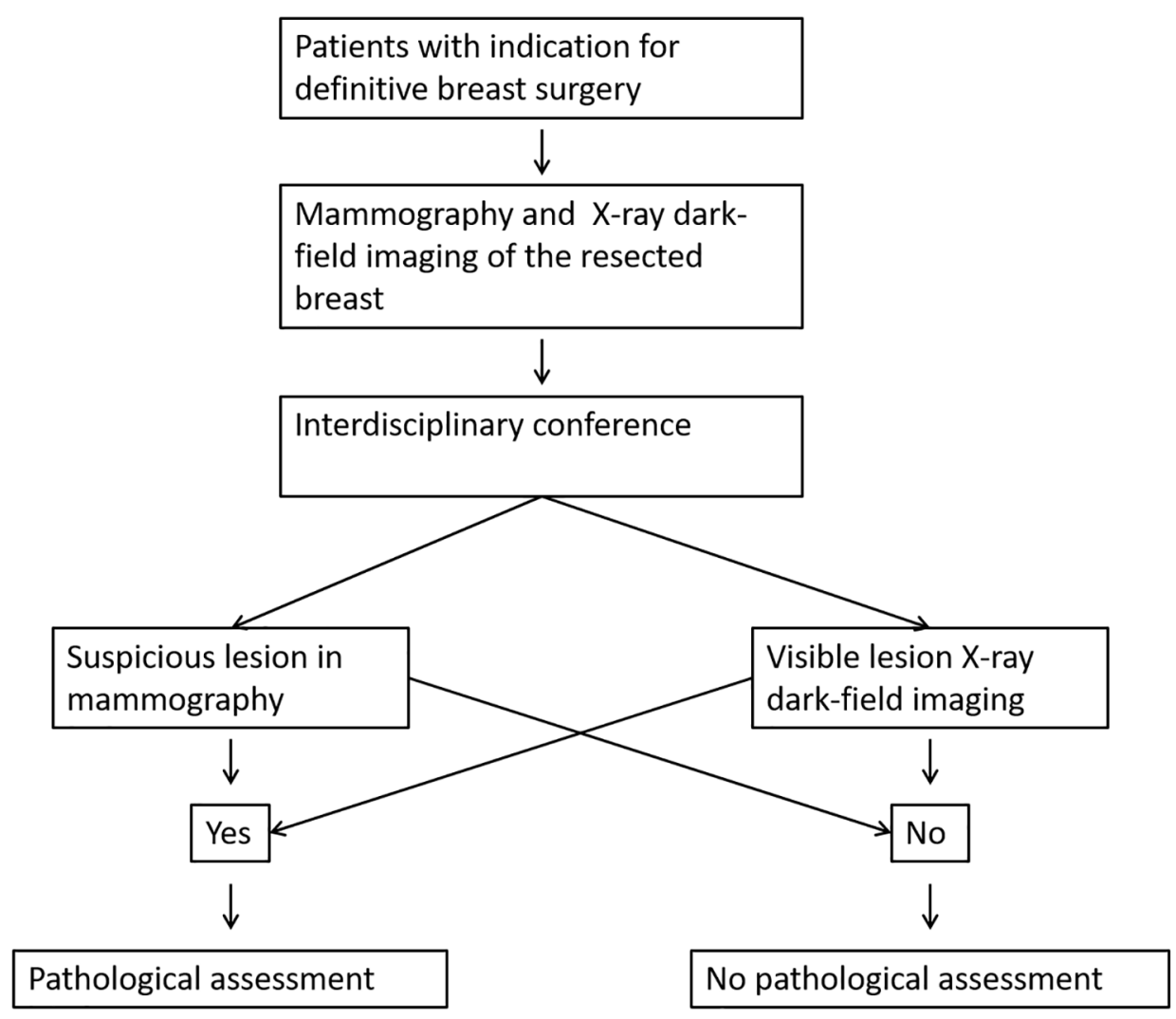

Figure 1. Overview of the imaging process, invasive procedures, and pathological assessment. (a) The patient has an indication for definitive breast surgery and provides informed consent for additional imaging and pathological assessment. (b) Performance of breast surgery and fixation of the specimen for imaging. (c) Performance of conventional mammographic and X-ray dark-field imaging. (d) Selection and marking of suspicious lesions at the interdisciplinary conference. (e) Histological preparation with pathological assessment of areas of interest from imaging. (f) Evaluation of the histological findings and radiologically suspicious lesions from both imaging techniques.

Individual breast-center pathologists carried out histological and immunohistochemical assessment, with confirmation of DCIS or invasive breast cancer, along with assessment of grading, estrogen-receptor (ER) status, progesterone receptor (PR) status, HER2 receptor status, and proliferation status (Ki-67), as described previously. ${ }^{20}$ The immunohistochemical evaluation of ER, PR, Ki-67, and HER2, and chromogene in situ hybridization of HER2, are quality controlled through the accreditation of the pathological laboratory (ISO/ DIN 17020, DAkkS) and were successfully validated by annual round-robin tests, as required by the German Cancer Society. All of the histopathological information used in the analysis was directly documented from the original pathology reports, which were reviewed by two investigators.

Matching the three modalities (mammography, $X$-ray dark-field imaging and pathology)

For the exact localization of the marked lesions by the pathologists, we merged the mammographic and X-ray dark-field images during a web-based conference. To achieve this in a first step, all lesions were marked with a circle on the X-ray dark-field image and the mammography. Every circle was numbered and listed in a database. Both images with the labeled lesions were stored. In a second step, both images were merged and printed out on an actual-size transparent (including labeled circles). In a third step, a standard syringe needle was pinned through the breastspecimen-covering transparent to re-identify the two-dimensional findings in the breast tissue. In a fourth step, the pathological examination was carried out in the needle-marked and labeled areas and the results were added to the database.

\section{Statistical considerations}

The data are presented descriptively. Patient and tumor data, as well as examination characteristics, are presented as counts and frequencies, means and standard deviation, or medians and range. 


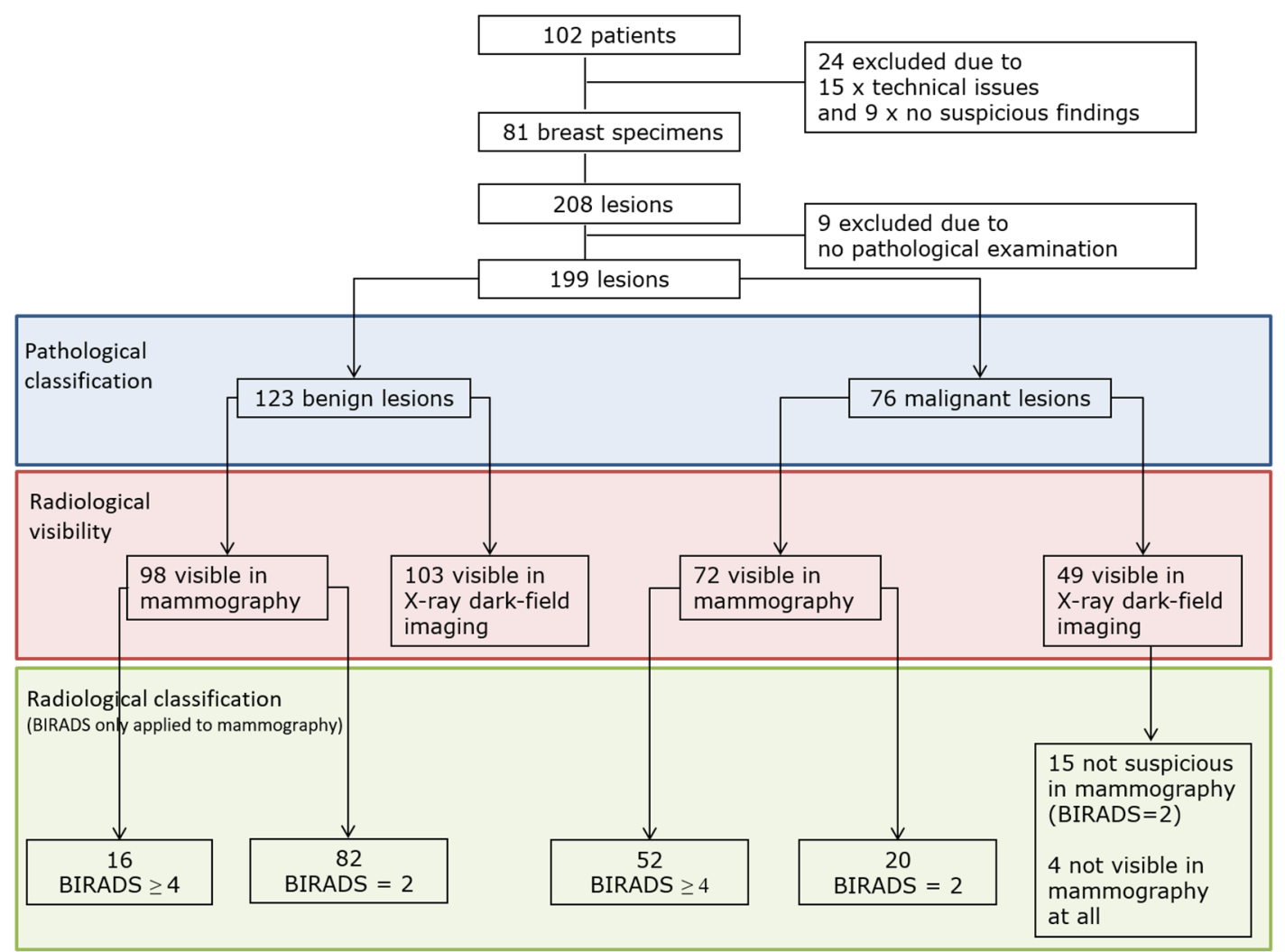

Figure 2. Overview of lesions that were suspicious on imaging and the corresponding histological results. of 208 marked lesions, 123 were histologically confirmed as benign, while 76 lesions were malignant leither breast cancer or ductal carcinoma in situ). The Breast Imaging Reporting and Data System (BI-RADS) classification was only applied to mammographic images. For detailed information about the individual lesions, please refer to Supplemental Table 1.

\section{Results}

\section{Patient and tumor characteristics}

A total of 102 patients were recruited from November 2013 to May 2015. A total of 24 of the initial 102 patients were excluded; 15 due to technical issues and 9 due to radiologically non-suspicious findings (Figure 2). The patients' mean age was 62 years (range $29-86$ years); $48 \%$ of them had a body mass index (BMI) $>25$ (Table 1). A total of 93 of the 102 patients presented with breast cancer, or had a history of breast cancer, or had confirmed breast cancer before neoadjuvant chemotherapy. The tumor characteristics are listed in Table 2. One patient underwent risk-reducing mastectomy and one patient, with breast cancer in one side, underwent contralateral prophylactic mastectomy. Suspicious lesions on imaging and corresponding histological results.

A total of 208 breast lesions were marked in 81 breast specimens, 9 of which were not pathologically assessed. In all, 123 lesions were histologically confirmed as benign; 98 of these were identified on mammography, 16 of which were classified as BI-RADS $\geqslant 4$ and 82 as BI-RADS 2 . Of these 123 lesions, 103 were visible on X-ray dark-field imaging. In all, 78 benign lesions were seen with both imaging modalities.

Seventy-six lesions were histologically confirmed as malignant. Of these, 72 were visible on mammography, 52 of which were classified as BI-RADS $\geqslant 4$, and 20 were classified as BI-RADS 2. In addition, 49 malignant lesions were visible on X-ray dark-field imaging, 15 of which had not been suspicious on mammography (BI-RADS 2). Four lesions that were seen on X-ray dark-field imaging had not been detected on mammography at all. Neither all malignant lesions nor all benign lesions were identifiable with each imaging technique alone (Figure 2). Out of these 76 lesions 13 were DCIS, 34 were no special type breast cancers (NSTs), 22 were invasive lobular carcinomas (ILCs) and 7 other breast cancer subtypes (Supplemental Table 1). 
Table 1. Patient characteristics.

\begin{tabular}{lll}
\hline & n or mean & \% or SD \\
\hline Patients ( $n)$ & 102 & \\
Age at diagnosis (mean) & 62 & \\
Menopausal status at diagnosis & & 73.5 \\
Postmenopausal & 75 & 26.5 \\
Premenopausal & 27 & \\
Age & 13 & 12.7 \\
$<45$ & 19 & 18.7 \\
$45-54$ & 70 & 68.6 \\
$>54$ & & 52 \\
BMI & 53 & 48 \\
$\quad \leqslant 25$ & 49 & \\
Patients with breast cancer & 93 & \\
\hline BMI, body mass index; SD, standard deviation. & & \\
\hline
\end{tabular}

Figure 3 shows an example of combined imaging with mammography and X-ray dark-field imaging. All four images show the same mastectomy specimen from a patient with breast cancer. The patient had undergone primary breast-conserving therapy, during which the tumor was not completely resected. A secondary mastectomy was therefore performed. The tumor bed was marked with three metal clips in the initial operation, and the round metal sphere was used to mark the nipple. The mammography images are shown on the left, displayed as inverted images for better comparability with the X-ray dark-field images on the right. The two images at the bottom show magnified views of the region of interest. The lesion marked with the arrow was only visible on X-ray dark-field imaging, but not on mammography. The pathological examination showed that it was an invasive lobular breast cancer.

\section{Discussion}

This study shows that adding X-ray dark-field imaging to conventional specimen mammography was able to highlight 15 additional malignant lesions that had been identified as lesions on specimen mammography alone, but had not been
Table 2. Tumor characteristics.

\begin{tabular}{|c|c|c|}
\hline & $n$ or mean & $\%$ or SD \\
\hline \multicolumn{3}{|l|}{ Tumor size } \\
\hline$\leqslant 2 \mathrm{~cm}$ & 42 & 45.2 \\
\hline$>2 \mathrm{~cm}$ & 51 & 54.8 \\
\hline \multicolumn{3}{|c|}{ Histological grade } \\
\hline 1 or 2 & 62 & 66.7 \\
\hline 3 & 31 & 33.3 \\
\hline \multicolumn{3}{|c|}{ Estrogen-receptor status } \\
\hline Positive & 61 & 65.6 \\
\hline Negative & 32 & 34.4 \\
\hline \multicolumn{3}{|c|}{ Nodal status } \\
\hline Positive & 25 & 26.9 \\
\hline Negative & 68 & 73.1 \\
\hline \multicolumn{3}{|c|}{ Neoadjuvant chemotherapy } \\
\hline No & 68 & 73.1 \\
\hline Yes & 25 & 26.9 \\
\hline \multicolumn{3}{|c|}{ Invasive breast cancer } \\
\hline No & 12 & 12.9 \\
\hline Yes & 81 & 87.1 \\
\hline
\end{tabular}

Histological tumor type

\begin{tabular}{lll} 
Ductal & 49 & 52.7 \\
Lobular & 15 & 16.1 \\
Ductal/lobular & 6 & 6.5 \\
Other & 23 & 24.7 \\
\hline
\end{tabular}

SD, standard deviation.

classified as suspicious. Moreover, four lesions were not visible on specimen mammography at all. On the one hand, adding X-ray dark-field imaging thus made it possible to identify 19 of 76 malignant lesions (25\%) that would have been missed with conventional specimen mammography alone. On the other hand, 27 lesions would have been missed with dark-field imaging alone.

A few studies to date have investigated potential practical applications for different methods of 
phase-contrast imaging, with an emphasis on increasing soft-tissue contrast, and improving the resolution of soft tissue, as well as microcalcifications. ${ }^{13-16,19,21-23}$ The first in vivo studies of phasecontrast imaging reported increased sensitivity and specificity in patients with lesions for which the malignancy status was initially uncertain. However, it has not yet been finally clarified whether the improvement in the imaging quality is dependent on the phase-contrast shift alone, or whether additional factors involving X-ray radiation are of importance. ${ }^{24-26}$

The published data on the application of X-ray dark-field imaging in breast diagnosis are still limited. In clinical trials, the method has been shown to improve the detection of microcalcifications in vivo. ${ }^{27}$ However, a large Japanese trial investigating nearly 4000 women with phase-contrast mammography did not show greater sensitivity or specificity in comparison with conventional specimen mammography. ${ }^{28}$

Grating-based interferometry has also been studied in preclinical settings in mastectomy specimens. ${ }^{13,14,16,22,23,29}$ In a study of 33 mastectomy specimens, phase-contrast imaging yielded increased imaging quality, sharpness, and detection of localized lesions. ${ }^{22}$ Another study with breast cancer specimens postulated that some tumors associated with very fine microcalcifications are not visible on standard specimen mammography, but can be detected with additional X-ray dark-field imaging. ${ }^{14}$ In a study by Anton et al., extremely small microcalcifications $3-30 \mu \mathrm{m}$ in size showed an especially intense interferometric signal in a set of six unembedded mastectomy specimens. ${ }^{13}$ Interestingly, Scherer et al. demonstrated that it was the micromorphology, rather than the chemical composition, of microcalcification clusters that determines their absorption and small-angle scattering, indicating that malignant or benign status depends on the microtexture. ${ }^{16}$

This study confirms that quite small microcalcifications that would otherwise have been missed can be identified with X-ray dark-field imaging. However, further analyses to elucidate the morphologic texture of the microcalcifications, as described by Scherer et al. ${ }^{16}$ were not carried out. Histological analyses of the relevant microcalcified lesions to confirm malignant or benign status were performed. Whether there is any connection between malignant lesions that are only detected
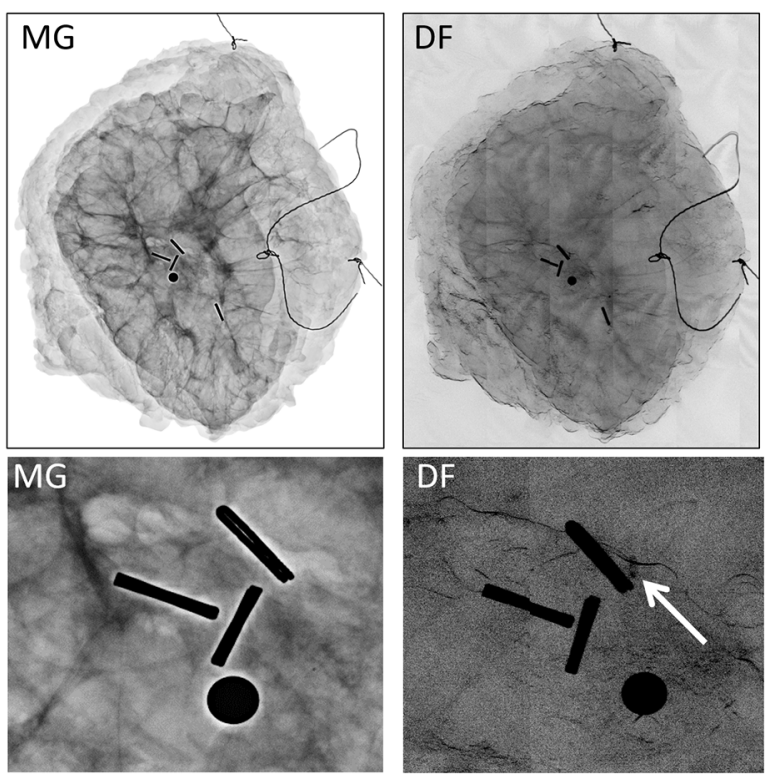

Figure 3. Representative image sets of combined mammography and X-ray dark-field imaging.

Left: inverted mammographic imaging; right: X-ray dark-field imaging. The white arrow shows a tumor-associated microcalcification that is visible on $\mathrm{X}$-ray dark-field imaging, but not on mammography. All four images show the same mastectomy specimen from a patient with breast cancer. The patient had undergone primary breast-conserving therapy, during which the tumor was not completely resected. A secondary mastectomy was therefore performed. The tumor bed was marked with three metal clips in the initial operation, and the round metal sphere was used to mark the nipple. The mammography images are shown on the left, displayed as inverted images for better comparability with the X-ray dark-field images on the right. The two images at the bottom show magnified views of the region of interest. The lesion marked with the arrow was only visible on X-ray dark-field imaging, but not on mammography. The pathological examination showed that it was an invasive lobular breast cancer.

DF, X-ray dark-field imaging; MG, mammography.

with X-ray dark-field imaging and the histological type of breast cancer was also investigated.

Fifteen lesions that were visible but not suspicious (BI-RADS 2) on specimen mammography were marked for histopathological examination because they were visible on X-ray dark-field imaging. Notably, six of these lesions were ILCs. In addition, two of the four lesions that were exclusively identified on X-ray dark-field imaging were also ILCs. Since lobular carcinoma is difficult to detect with mammography, ${ }^{30} \mathrm{X}$-ray darkfield imaging may be of particular interest for this type of cancer. Due to the small sample size, however, it is not possible to draw any conclusions regarding a connection between detection on X-ray dark-field imaging and histological type.

The present study has some limitations. The majority of the patients included underwent 
breast surgery due to breast cancer. Therefore, the probability of findings is higher than in a normal screening population. The study includes a larger number of women who underwent mastectomy, which might have led to a selection bias in favor of patients with higher tumor stages and with prior treatment. Some of the smaller lesions detected that were marked due to X-ray darkfield imaging were satellite lesions. However, the main lesions were detected with specimen mammography in all patients with breast cancer. Consequently, although X-ray dark-field imaging identified additional breast cancer lesions, specimen mammography alone did not miss any cases of breast cancer.

The simple addition of X-ray dark-field imaging without classifying the X-ray dark-field lesions as benign or malignant leads to a decrease in specificity. Therefore, further criteria for classification of X-ray dark-field lesions must be established and evaluated.

To avoid interobserver bias, both the mammographic and X-ray dark-field images were assessed by two independent radiologists and were finally discussed at an interdisciplinary conference. Nevertheless, selection bias cannot be ruled out. Finally, the sample size would need to be larger in order to draw further conclusions - for example, with regard to connections between the histological type and better detection with one or another imaging method. To the best of our knowledge, this is the largest dataset yet published investigating the efficacy of conventional specimen mammography and the addition of X-ray dark-field imaging in breast specimens.

$\mathrm{X}$-ray dark-field imaging may be able to provide more accurate and detailed radiological classification of suspicious breast lesions. Adding X-ray dark-field imaging may increase the detection rate and improve preoperative planning in deciding between mastectomy or breast-conserving therapy, particularly in patients with invasive lobular breast cancer.

The workflow employed in this study is not suitable for everyday clinical use. In the next step, the interferometer will need to be included in a commercial system, so that normal breast compression and patient positioning can be used. In addition, the normal absorption image quality available with interferometric imaging will require suitable postprocessing in order to achieve the quality of state-of-the-art mammography. The image information obtained from dark-field imaging could be fused into absorption images in order to further speed up the reading process.

Since some of the lesions that were only detected on X-ray dark-field imaging were benign, efforts will need to be made to find out what causes interferometric signals, in order to improve the specificity. Future studies should also investigate larger numbers of samples, with an emphasis on differences in the detection of histological tumor types, types of calcification (hydroxyapatite versus calcium oxalate), and breast density and size.

In addition, it is to mention that the used X-ray dark-field system until now is not ready for in vivo clinical use as the exposure time, dosage and handling is not established.

In conclusion, this study shows that adding X-ray dark-field imaging to specimen mammography can identify additional malignant lesions that are not suspicious or not visible with mammography alone. These findings should be investigated in larger trials.

\section{Conflict of interest statement}

$M R$ is an employee of Siemens Healthcare $\mathrm{GmbH}$, Erlangen, Germany. All other authors declare that they do not have any conflicts of interest.

\section{Funding}

The authors disclose receipt of the following financial support for the research, authorship, and/or publication of this article: this project was funded by the Spitzencluster Initiative of the German Ministry for Education and Science $(\mathrm{BMBF})$, with the grant number $13 \mathrm{EX} 1212 \mathrm{C}$, subproject BD14.

\section{Supplemental material}

Supplemental material for this article is available online.

\section{References}

1. Kelly KM, Dean J, Comulada WS, et al. Breast cancer detection using automated whole breast ultrasound and mammography in radiographically dense breasts. Eur Radiol 2010; 20: 734-742.

2. Nelson HD, Tyne K, Naik A, et al. Screening for Breast Cancer: Systematic Evidence Review Update 
for the U.S. Preventive Services Task Force (Evidence Review Update No. 74). Rockville, MD: Agency for Healthcare Research and Quality, 2009.

3. Wockel A, Festl J, Stuber T, et al.

Interdisciplinary screening, diagnosis, therapy and follow-up of breast cancer. Guideline of the DGGG and the DKG (S3-Level, AWMF Registry Number 032/045OL, December 2017) - Part 1 with recommendations for the screening, diagnosis and therapy of breast cancer. Geburtshilfe Frauenheilkd 2018; 78: 927-948.

4. Luiten JD, Voogd AC, Luiten EJT, et al. Trends in incidence and tumour grade in screen-detected ductal carcinoma in situ and invasive breast cancer. Breast Cancer Res Treat 2017; 166: 307-314.

5. Bluekens AM, Holland R, Karssemeijer N, et al. Comparison of digital screening mammography and screen-film mammography in the early detection of clinically relevant cancers: a multicenter study. Radiology 2012; 265: $707-714$.

6. Atasoy MM, Tasali N, Cubuk R, et al. Vacuum-assisted stereotactic biopsy for isolated BI-RADS 4 microcalcifications: evaluation with histopathology and midterm follow-up results. Diagn Interv Radiol 2015; 21: 22-27.

7. Liberman L, Gougoutas CA, Zakowski MF, et al. Calcifications highly suggestive of malignancy: comparison of breast biopsy methods. $A \mathcal{F R} A m \mathcal{F}$ Roentgenol 2001; 177: 165-172.

8. Bent CK, Bassett LW, D’Orsi CJ, et al. The positive predictive value of BI-RADS microcalcification descriptors and final assessment categories. AFR Am $\mathcal{F}$ Roentgenol 2010; 194: 1378-1383.

9. Schulz-Wendtland R, Fuchsjager M, Wacker T, et al. Digital mammography: an update. Eur $\mathcal{F}$ Radiol 2009; 72: 258-265.

10. Elmore JG, Barton MB, Moceri VM, et al. Tenyear risk of false positive screening mammograms and clinical breast examinations. $N$ Engl $\mathcal{F ~ M e d ~}$ 998; 338: 1089-1096.

11. Evans A. Hormone replacement therapy and mammographic screening. Clin Radiol 2002; 57: 563-564.

12. Berg WA, Gutierrez L, NessAiver MS, et al. Diagnostic accuracy of mammography, clinical examination, US, and MR imaging in preoperative assessment of breast cancer. Radiology 2004; 233: 830-849.

13. Anton G, Bayer F, Beckmann MW, et al. Grating-based darkfield imaging of human breast tissue. Z Med Phys 2013; 23: 228-235.
14. Michel T, Rieger J, Anton G, et al. On a darkfield signal generated by micrometer-sized calcifications in phase-contrast mammography. Phys Med Biol 2013; 58: 2713-2732.

15. Pfeiffer F, Herzen J, Willner M, et al. Gratingbased X-ray phase contrast for biomedical imaging applications. $Z$ Med Phys 2013; 23: 176-185.

16. Scherer K, Braig E, Ehn S, et al. Improved diagnostics by assessing the micromorphology of breast calcifications via X-Ray dark-field radiography. Sci Rep 2016; 6: 36991.

17. Beckmann MW, Brucker C, Hanf V, et al. Quality assured health care in certified breast centers and improvement of the prognosis of breast cancer patients. Onkologie 2011; 34: 362-367.

18. Kowalski C, Ferencz J, Brucker SY, et al. Quality of care in breast cancer centers: results of benchmarking by the German Cancer Society and German Society for Breast Diseases. Breast 2015; 24: 118-123.

19. Keyrilainen J, Fernandez M, Fiedler S, et al. Visualisation of calcifications and thin collagen trands in human breast tumour specimens by the diffraction-enhanced imaging technique: a comparison with conventional mammography and histology. Eur F Radiol 2005; 53: 226-237.

20. Fasching PA, Heusinger K, Haeberle L, et al. $\mathrm{Ki67}$, chemotherapy response, and prognosis in breast cancer patients receiving neoadjuvant treatment. BMC Cancer 2011; 11: 486.

21. Fernandez M, Keyrilainen J, Serimaa R, et al. Human breast cancer in vitro: matching histopathology with small-angle X-ray scattering and diffraction enhanced X-ray imaging. Phys Med Biol 2005; 50: 2991-3006.

22. Hauser N, Wang Z, Kubik-Huch RA, et al. A study on mastectomy samples to evaluate breast imaging quality and potential clinical relevance of differential phase contrast mammography. Invest Radiol 2014; 49: 131-137.

23. Wang Z, Hauser N, Singer G, et al. Non-invasive classification of microcalcifications with phasecontrast X-ray mammography. Nat Commun 2014; 5: 3797.

24. Castelli E, Tonutti M, Arfelli F, et al. Mammography with synchrotron radiation: first clinical experience with phase-detection technique. Radiology 2011; 259: 684-694.

25. Arfelli F, Bonvicini V, Bravin A, et al. Mammography with synchrotron radiation: phase-detection techniques. Radiology 2000; 215 : 286-293. 
Visit SAGE journals online journals.sagepub.com/ home/tam

(3)SAGE journals
26. Williams IM, Siu KK, Gan R, et al. Towards the clinical application of $\mathrm{X}$-ray phase contrast maging. Eur F Radiol 2008; 68(3 Suppl.): S73-S77.

27. Tanaka T, Honda C, Matsuo S, et al. The first trial of phase contrast imaging for digital full-field mammography using a practical molybdenum X-ray tube. Invest Radiol 2005; 40: 385-396.

28. Morita T, Yamada M, Kano A, et al. A Comparison between film-screen mammography and full-field digital mammography utilizing phase contrast technology in breast cancer screening programs. In: Krupinski EA (ed.)
Proceedings on Digital mammography: 9th international workshop, IWDM 2008, Tucson, AZ, 20-23 July 2008. Berlin, Heidelberg: Springer Berlin Heidelberg, 2008, pp. 48-54.

29. Stampanoni $M$, Wang $Z$, Thuring $T$, et al. The first analysis and clinical evaluation of native breast tissue using differential phase-contrast mammography. Invest Radiol 2011; 46: 801-806.

30. Brem RF, Ioffe M, Rapelyea JA, et al. Invasive lobular carcinoma: detection with mammography, sonography, MRI, and breast-specific gamma imaging. Am f Roentgenol 2009; 192: 379-383. 